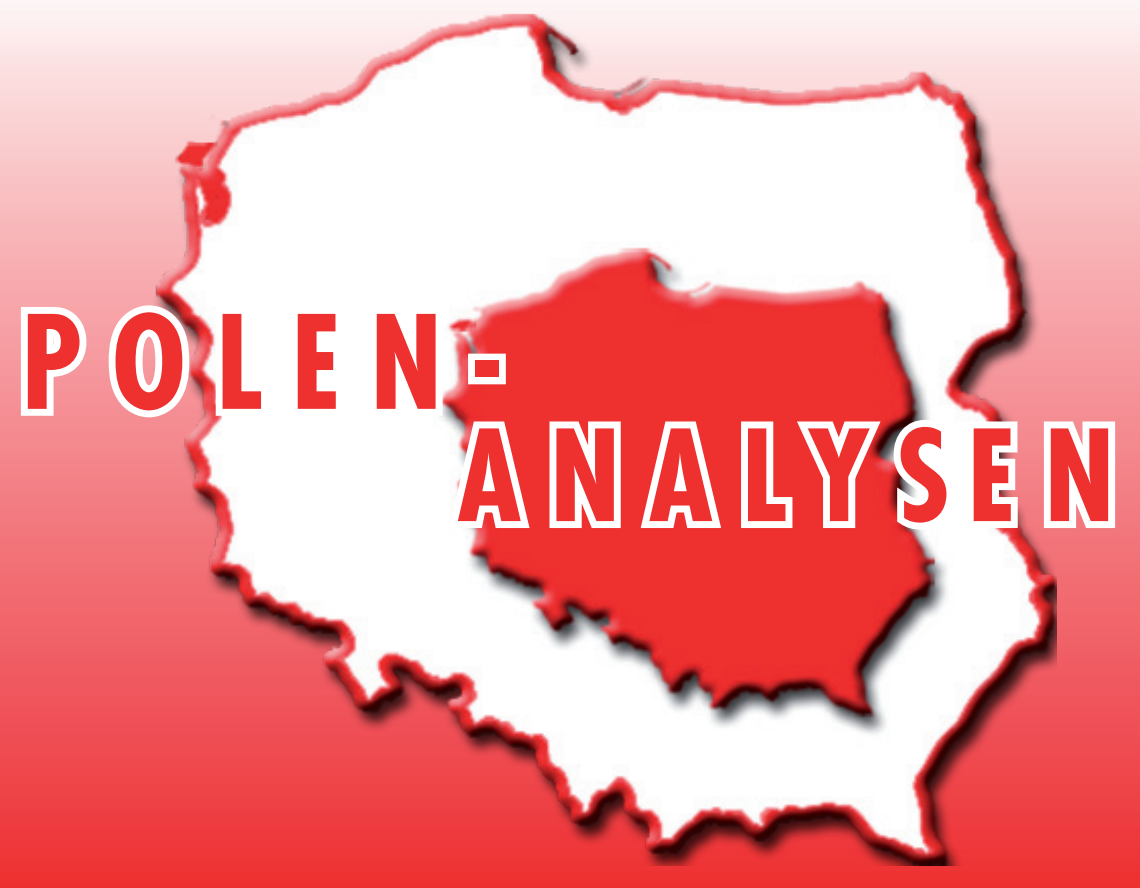

www.laender-analysen.de/polen

\title{
POLNISCHSPRACHIGE MIGRANTEN IN DEUTSCHLAND
}

ANALYSE

Zum Minderheitenstatus der polnischsprachigen Migranten in Deutschland Andrzej Kaluza, Darmstadt

DOKUMENTATION

Quellen zum Text

CHRONIK

Vom 18. bis zum 31. Oktober 2011 


\title{
Zum Minderheitenstatus der polnischsprachigen Migranten in Deutschland
}

\author{
Andrzej Kaluza, Darmstadt
}

\section{Zusammenfassung}

Der deutsch-polnische Nachbarschaftsvertrag von 1991 sichert Angehörigen der deutschen Minderheit in Polen und Angehörigen der Gruppe deutscher Staatsbürger mit polnischer Abstammung oder Bekenntnis zur polnischen Sprache, Kultur oder Tradition vergleichbare Rechte zu. Die etwa 300.000 polnischen Staatsbürger, die sich als Deutsche verstehen, werden nicht nur durch den bilateralen Vertrag, sondern auch durch den polnischen Gesetzgeber als Minderheit anerkannt und genießen dadurch bestimmte Förderrechte (Bildung, Kultur, Medien) von Seiten des Staates. Dagegen hat die polnischsprachige Gruppe in Deutschland formalrechtlich nicht den Status einer nationalen Minderheit, da sie nicht zu den traditionellen in Deutschland ansässigen Minderheiten zählt, sondern aus Migranten besteht. Vertreter der »Polonia«-Organisationen in Deutschland streben diesen Status dennoch an. Der Autor weist darauf hin, dass sowohl die historischen Argumente wie auch die Ausdifferenzierung der Selbstidentifikation der Angehörigen der polnischen Gruppe in Deutschland den Status einer nationalen Minderheit nicht begründen können, gleichwohl aus dem deutsch-polnischen Nachbarschaftsvertrag einzulösende Verpflichtungen (z. B. Förderung des Polnischen als Muttersprache) bestehen.

A s 20 Jahre nach Abschluss des deutsch-polnischen Vertrags über gute Nachbarschaft und freundschaftliche Zusammenarbeit vom 17. Juni 1991 an der überwiegend positiven Bilanzierung gearbeitet wurde, rückte das Scheitern einer gemeinsamen Erklärung beider Staaten zur Lage der sog. "polnischen Gruppe/Minderheit« in Deutschland in den Vordergrund. Bereits seit Frühjahr 2010 waren drei Arbeitsgruppen beauftragt, Forderungen von Organisationen der "Polonia $"{ }^{*}$ in Deutschland zu identifizieren und Möglichkeiten ihrer Erfüllung zu überprüfen. Dass damals Vertreter dieser Verbände mit am Tisch saßen, werteten diese als ihren großen Erfolg. Bisher waren sie nämlich von den Behörden hinsichtlich ihrer Seriosität und Professionalität nicht selten mit Argwohn beäugt worden.

Die »Polonia«-Organisationen setzten sich gleich mehrere Ziele. Kurzfristig, im Vorfeld der Vertragsevaluierung, ging es um symbolische Politik: die Rehabilitierung der »Polonia«-Vertreter, die nach 1939 Repressalien des NS-Regimes ausgesetzt gewesen und in deutschen Konzentrationslagern als politische Gefangene misshandelt worden waren (etwa 1.200 Aktivisten hatten so ihr Leben verloren), die Entschädigung für das vom NSStaat beschlagnahmte Vermögen der polnischen Organisationen (Banken, Gewerkschaftskassen, Schulen), die Stärkung der kulturellen und sprachlichen Identität der in Deutschland lebenden Polen durch mehr für diese Zwecke bereitgestellte Finanzmittel sowie die Einrichtung eines Verbindungsbüros der »Polonia«-Organisationen in Berlin, das die Aktivitäten der einzelnen

\footnotetext{
Als »Polonia" werden im Ausland lebende polnische Auswanderer und ihre Nachkommen bezeichnet. Dabei unterscheidet man oft nicht zwischen der Gesamtheit der Gruppe und den politischen Organisationen, die die Erhaltung einer als national, kulturell und religiös homogen definierten "polnischen Identität« anstreben.
}

Verbände koordinieren und unterstützen soll. Alle diese Forderungen wurden im Laufe der Gespräche von der deutschen Seite aufgegriffen; eine große, nicht nur symbolische Bedeutung hatte dabei die Entschließung des Bundestags vom 10. Juni 2011, in der all jene Punkte genannt sind (s. Quelle 1 im Anhang). Darüber hinaus wird in Bochum ein Dokumentationszentrum für die Geschichte der »Polonia» in Deutschland eingerichtet.

Allerdings ging es noch um ein weit wichtigeres Ziel, das nicht erreicht werden konnte und das letztlich zum Eklat führte: die Zuerkennung eines Minderheitenstatus für die polnische Gruppe in Deutschland. Mit dem "Status« wäre die »Asymmetrie in der Definition« beider Volksgruppen, wie sie im Vertrag vorgenommen wurde, vom Tisch, denn der Vertrag spricht nur von einer deutschen Minderheit in Polen, nicht aber von einer polnischen Minderheit in Deutschland, so die "Polonia»-Vertreter. Sie hofften auf eine definitorische Gleichstellung seitens der Vertragspartner und verwiesen auf verschiedene Argumente, die diese Forderung untermauern sollten. Nur dann, so ihre einhellige Meinung, könnten die erhobenen Ansprüche auf eine aktive Förderung der Kultur und Muttersprache der in Deutschland lebenden Polen rechtlich verankert und dadurch vor Gerichten einklagbar werden. Auf diese Weise hätte u. a. die ihrer Meinung nach »skandalöse" und "willkürliche« Vergabepraxis finanzieller Mittel der deutschen Behörden gegenüber den »Polonia«-Organisationen ein Ende. Die Erlangung des Minderheitenstatus müsste demnach eine ganz andere finanzielle Ausstattung der Organisationen nach sich ziehen, die der Stärkung der sprachlichen und kulturellen Identität von ca. 1-2 Mio. in Deutschland lebenden Polen (nach der Definition der "Polonia«-Vertreter) zugute käme. Die gegenwärtige Regelung, nach der von deutscher Seite eine Summe von etwa 1,2 Mio. Euro 
für muttersprachlichen Unterricht (vorwiegend in Nordrhein-Westfalen und Rheinland-Pfalz) und etwa 300.000 Euro (vom Bundesbeauftragten für Kultur und Medien/ BKM) zur Stärkung der kulturellen Identität zur Verfügung gestellt werden, wurde als viel zu niedrig abgelehnt. Zum Vergleich wies man dabei auf die in Polen lebenden Deutschen (ca. 200.000-300.000) hin, die für kulturelle Projekte, Sprachunterricht und Medien vom polnischen Staat Zuwendungen in Höhe von ca. 80 Mio. PLN (ca. 20 Mio. Euro) erhalten. So begann eine zermürbende und frustrierende Schlacht um den Minderheitenstatus.

\section{Auf der Suche nach einem "verlorenen" Minderheitenstatus}

Wie erwähnt, definiert der deutsch-polnische Nachbarschaftsvertrag von 1991 nur die Deutschen in Polen als eine "nationale Minderheit«. Darüber hinaus kommen diese in den Genuss des 2005 in Polen beschlossenen Minderheitengesetzes, das ihnen positive Förderrechte gewährt (ähnlich wie auch anderen nationalen Minderheiten, etwa den Ukrainern oder den Litauern). Auf der anderen Seite spricht der Vertrag in Artikel 20 von »Personen deutscher Staatsangehörigkeit, die polnischer Abstammung sind oder die sich zur polnischen Sprache, Kultur, Tradition bekennen«. In vielen Interpretationen wird daraus eine "faktische Gleichstellung" beider Gruppen abgeleitet; von den Vertretern der »Polonia" wird dies jedoch in Hinblick auf die ihrer Meinung nach nicht transparente Vergabepraxis der Mittel durch die deutsche Seite immer wieder in Zweifel gezogen.

Für die »Polonia«-Organisationen und die sie unterstützenden polnischen Politiker und Medien stand seit Beginn der Verhandlungen außer Diskussion, dass nur die »Wiedererlangung« des rechtlichen Minderheitenstatus der Polen in Deutschland eine Änderung der Lage bringen würde. Dabei wurde auf die Geschichte verwiesen, die Argumente für die gegenwärtigen Ansprüche liefern sollte. Laut diesen Auffassungen steht außer Zweifel, dass die Polen in Deutschland bereits vor dem Zweiten Weltkrieg den Status einer "nationalen Minderheit« genossen haben, der ihnen 1940 durch das nationalsozialistische Deutschland entzogen worden sei. Diese Argumentation, die auch von den Autoren eines vom polnischen Außenministerium in Auftrag gegebenen Gutachtens unterstützt wurde (in dem betreffenden Teil findet sich keine rechtsgeschichtliche Analyse des Sachverhalts, sondern nur eine einzige Feststellung: "Nach dem Ersten Weltkrieg saßen im Jahr 1922 Vertreter der polnischen Minderheit, die von der Weimarer Republik offiziell anerkannt wurde, in 283 Kommunalparlamenten.»), machten sich auch viele polnische Politiker, ja sogar polnische wie deutsche Medien zu eigen. Selbst die Analyse des Instituts für Auslandsbeziehungen (ifa)
"Zwischen zwei Welten« von Sebastian Nagel (2009), die im Auftrag des BKM entstand, spricht in dem Kontext vom "Entzug des bis dato staatlich anerkannten Minderheitenstatus«. Diese Behauptung ist jedoch nicht belegt.

Die historischen Ansprüche der "Polonia" auf den Minderheitenstatus scheinen bei näherem Hinsehen diffus. Die rechtliche Lage ist unsicher und erlaubt es nicht, Zuwendungsansprüche in der Gegenwart zu stellen.

Auch wenn der Versailler Vertrag vom besiegten Deutschland allgemein Minderheitenschutz verlangte, hat die Weimarer Republik keine explizite Minderheitengesetzgebung auf den Weg gebracht. Im Juristendeutsch hießen die Vertreter der in Deutschland ansässigen Völker auch nicht Minderheiten, sondern »fremdsprachige Volksteile«. Die Weimarer Verfassung erwähnte sie in einem einzigen Artikel: »Die fremdsprachigen Volksteile des Reiches dürfen durch die Gesetzgebung und Verwaltung nicht in ihrer freien, volkstümlichen Entwicklung, besonders im Gebrauch der Muttersprache beim Unterricht, sowie bei der inneren Verwaltung und der Rechtspflege beeinträchtigt werden« (Art. 113), dem aber keine Ausführungsbestimmungen folgten. Folglich handelte es sich dabei um eine deklaratorische Absicht, wie sie in grundsätzlichen Rechtsakten wie Verfassungen üblich ist, nicht um ein unmittelbares Minderheitenrecht. Die negative Formulierung bringt einen allgemeinen Schutzgedanken zum Ausdruck, der besagt, dass der Staat zwar allgemeine Gruppenrechte gewähren, sich aber aus deren aktiver Gestaltung heraushalten soll. Vertreter national definierter Gruppen konnten sich auf diese Weise in der Weimarer Republik auf der Basis des allgemeinen Vereinsrechts autonom entwickeln; eine Verpflichtung des Staates, sie in ihren kulturellen Bedürfnissen zu fördern, resultierte daraus nicht. Dementsprechend deutlich sind die Worte von Jan Kaczmarek, dem Generalsekretär und Vordenker des Bundes der Polen in Deutschland, in seiner Zeitschrift »Kulturwehr« von 1929 zur deutschen Minderheitengesetzgebung: "Und eben das Fehlen von Ausführungsbestimmungen macht den Artikel (Art. 113 der Reichsverfassung - Anm. AK) leblos, macht ihn zum 'Fetzen Papier . Noch bedeutungsloser wird der Artikel durch seine negative Feststellung sdarf nicht beeinträchtigt werden anstatt des unbedingt notwendigen `muss gefördert werden`."

Nur auf dieser Rechtsgrundlage entstand 1922 der Bund der Polen, die bedeutendste polnische Organisation in der Zwischenkriegszeit, deren mitgliederstärkste Region der deutsche Teil Oberschlesiens war. Nach der Teilung Oberschlesiens im Jahre 1922 bestand dort laut einem zwischen Deutschland und Polen für 15 Jahre in Genf geschlossenen Vertrag ein garantiertes Minderheitenrecht, das auch staatliche Zuschüsse für den muttersprachlichen Unterricht vorsah. Dies wurde in der Pra- 
xis von der deutschen Seite nur zögerlich gewährt und galt eben nicht für ganz Deutschland.

In jüngster Zeit verweisen einige Diskussionsteilnehmer auf zwei Dokumente aus der Zeit des Nationalsozialimus, die die Statusfrage untermauern sollen. Bevor der auf Gegenseitigkeit beruhende Minderheitenschutz in Oberschlesien mit dem Erlöschen des Genfer Vertrags 1937 außer Kraft gesetzt werden sollte, bemühten sich beide Regierungen um eine neue Regelung. Die Intention der deutschen Seite war es, auf diese Weise die Rechte der deutschen Gruppe in Polen (vor allem in Oberschlesien) zu garantieren. Das erklärt auch das aktive Bemühen der Nationalsozialisten um eine solche Vereinbarung mit Polen. Im Wortlaut der Übereinstimmenden Erklärung sucht man vergeblich nach Vereinbarungen, die eine aktive Förderung der polnischen Minderheit im nationalsozialistischen Deutschland bestätigen würden (s. Quelle 2). Auf einem anderen Blatt steht, dass diese Vereinbarung praktisch nicht in Kraft trat, ganz im Gegenteil, der Druck auf die polnischen Organisationen stieg kontinuierlich bis zum Beginn des Zweiten Weltkrieges 1939.

Im Sommer 2010 verlangte der Berliner Anwalt Stefan Hambura in einem Brief an Bundeskanzlerin Angela Merkel, das formelle Verbot der polnischen Organisationen in Deutschland aus dem Jahr 1940 durch ein explizites Gesetz im Vorfeld des 20. Jahrestages des deutsch-polnischen Vertrags und des 70. Jahrestages des Weltkriegsausbruchs zurückzunehmen. Hambura verwies dabei auf eine "enorme symbolische Bedeutung" einer solchen Regelung. Er veröffentlichte eine bis dato kaum beachtete Verordnung (s. Quelle 3) vom 27. Februar 1940, die seiner Auffassung den Anspruch auf den Minderheitenstatus begründete. Die Veröffentlichung brachte die Diskussion - zumal unter einigen polnischen Politikern - neu ins Rollen. Abgesehen davon, dass das Hauptanliegen des Briefes ein rechtliches Eigentor war (das Bundesministerium der Justiz antwortete sinngemäß, dass alle Unrechtsakte des nationalsozialistischen Regimes mit dem Inkrafttreten des Grundgesetzes 1949 automatisch ungültig geworden sind, wenn sie inhaltlich im Widerspruch zum GG standen; die Aufhebung des Inhalts der besagten Regelung bedürfe also keines eigenen Aktes), fand sich darin auch kein Hinweis auf die rechtliche Aberkennung eines wie auch immer verbrieften Minderheitenstatus. Auch dieses Dokument spricht lediglich von "Organisationen der polnischen Volksgruppe«.

Die historischen Argumente sehen demnach dürftig aus: Außer einem allgemeinen Minderheitenschutz im Art. 113 der Reichsverfassung gab es in der Vorkriegszeit keine Rechtsakte, die den Polen in Deutschland den Status einer anerkannten Minderheit garantiert hätten. Die bloße Existenz von Organisationen darf hier nicht mit dem rechtlichen Status einer Volksgruppe verwechselt werden. Hinzu kommt, dass sich der Gedanke, eigene nationale Minderheiten aktiv in ihrer Identität zu fördern, erst nach dem Zweiten Weltkrieg völkerrechtlich durchgesetzt hatte.

Die Fixierung der »Polonia«-Organisationen auf die Statusfrage resultiert z. T. auch aus der Wahrnehmung der positiv formulierten Rechte der in Deutschland nach 1949 anerkannten nationalen Minderheiten, denn diese verpflichten den Staat (in der Regel die betroffenen Bundesländer), erhebliche finanzielle Zuschüsse zu gewähren, um die sprachliche und kulturelle Identität der Mitglieder dieser Gruppen zu fördern. In den Genuss dieser Mittel kommen aber ausschließlich Volksgruppen, die völkerrechtliche Kriterien erfüllen: Es handelt sich dabei um Gruppen von Menschen, die eine eigene nichtdeutsche Identität besitzen, gleichzeitig aber das Gebiet des deutschen Staates seit Generationen bewohnen. So haben z. B. die Dänen Minderheitenrechte, die ihnen ein deutsch-dänisches Abkommen von 1955 gewährt, die aber zumeist nur auf wenige Landkreise in Schleswig-Holstein beschränkt sind; ein aus Flensburg stammender Deutscher dänischer Herkunft kann dort einen dänischsprachigen Kindergarten für seine Nachkommen verlangen, nicht aber in Berlin. Ähnlich verhält es sich mit der Aufhebung der 5\%-Hürde für politische Parteien, die für die Dänen nur in Schleswig-Holstein gilt.

Nach dieser Definition wären die Polen im Deutschland der Zwischenkriegszeit (insbesondere Oberschlesien) sicherlich auch als »Minderheit« zu fassen; die nach dem Krieg vorgenommenen Grenzänderungen schließen jedoch die territoriale Kontinuität, die damals gegeben war (Oberschlesien, Ostpreußen), aus. Da man annehmen muss, dass es die »Ruhr-Polen« nicht mehr als national definierte Gruppe gibt, gelten die nach 1945 aus Polen nach Deutschland eingewanderten Menschen hierzulande als Migranten. In der Frage des nationalen Minderheitenstatus, der bei den bilateralen Gesprächen 2010-2011 von »Polonia«-Organisationen (nicht von der polnischen Regierung!) gefordert wurde, konnten sich diese (trotz mehrerer "Versprecher" deutscher Politiker, die in der Öffentlichkeit von einer "polnischen Minderheit" sprachen) gegen die deutsche Rechtsauffassung demnach nicht durchsetzen. Dahinter standen neben den rechtlichen Gründen auch Befürchtungen, ein Präzedenzfall werde seitens zahlreicher Einwanderergruppen finanzielle Forderungen an den deutschen Staat hervorrufen. Übrigens gelten auch in anderen Ländern, in denen polnische Migranten leben, ähnliche Auffassungen: In keinem Land Westeuropas genießen eingewanderte Polen Minderheitenrechte, viele Länder (Frankreich, Großbritannien, USA) haben gar keine Minderheitengesetzgebung. Auch das Argument der "fehlenden Symmetrie» zieht in diesem Fall nicht, 
da Minderheiten objektiv vorhanden sind und nicht dadurch entstehen, dass ein anderer Staat (hier Polen) einem Teil seiner Bürger bestimmte Rechte gewährt. Die starke Fokussierung der Diskussion auf die Minderheitenfrage stellte sich als Sackgasse heraus.

\section{Die polnische Gruppe in Deutschland - wen repräsentieren die "Polonia«-Organisationen?}

Da die historischen Ansprüche nicht ganz überzeugen, bemühten sich Vertreter der "Polonia", auch die hohe Zahl und die Aktivität der in Deutschland lebenden Polen als Argument für den Minderheitenstatus anzuführen. Hier ist wieder ein Rückgriff auf die oben genannte Definition im Vertrag von 1991 nützlich, da sie einen überwiegenden Teil der Migranten aus Polen, die heute deutsche Staatsbürger sind, umfasst. Es sind in der Regel Aussiedler und Spätaussiedler. Die meisten von ihnen haben Polen nach 1980 verlassen und besitzen heute de facto die doppelte (deutsche und polnische) Staatsbürgerschaft. Auswanderer, die das Land offiziell auf Antrag im Rahmen deutsch-polnischer Vereinbarungen (Familienzusammenführung) bis etwa 1982 verlassen haben, mussten dagegen bei der Ausreise auf ihre polnische Staatsbürgerschaft verzichten. In Deutschland leben heute aber auch ca. 400.000 Polen mit ausschließlich polnischem Pass. Sie sind von den Regelungen des Vertrags nicht direkt betroffen.

Den Löwenanteil der ca. 1,5 Mio. Menschen zählenden Gruppe stellen die Aussiedler bzw. die Spätaussiedler dar, die seit den 1950er Jahren in beide deutsche Staaten in der Regel freiwillig ausgewandert sind und ihre deutsche Volkszugehörigkeit in einem formellen Verfahren nachgewiesen haben. Darüber hinaus gehören dieser Gruppe polnische politische Emigranten aus der »Solidarność«-Zeit an (nach 1981 als anerkannte politische Flüchtlinge und De-facto-Flüchtlinge) sowie andere Personen aus Polen, die einen Aufenthaltstitel in der Bundesrepublik erhalten und im Laufe der Zeit die deutsche Staatsangehörigkeit angenommen haben (z. B. Ehepartner in binationalen Ehen).

Tatsächlich kamen die Migranten aus Polen nach Deutschland nach dem Zweiten Weltkrieg in mehreren "Wellen«. Dabei muss man eine gewisse Zahl von Polen berücksichtigen, die sich 1945 im Zuge des Kriegsgeschehens (Zwangsarbeiter, KZ-Insassen, Soldaten der alliierten Armeen) in Deutschland befunden und später zu einem (kleinen) Teil sogar niedergelassen hat. Nach der Zeit von Flucht (1944-1945) und Vertreibung (19451947) blieben in Polen zunächst nur noch Gruppen von Deutschen sowie eine relativ große Gruppe von sog. "Autochthonen", d. h. einer in der Regel zweisprachigen Grenzbevölkerung, Menschen, die nach Art. 116 GG weiterhin deutsche Staatsbürger waren, in der offiziellen polnischen Lesart aber gleichzeitig als "germanisierte" Polen aufgefasst wurden und den Anspruch Polens auf die sog. "Wiedergewonnenen Gebiete« untermauern sollten. Aus diesen Kreisen rekrutierten sich Aussiedler und Spätaussiedler. Zwischen 1950 und 1979 emigrierten so aus Polen im Zuge der humanitär begründeten »Familienzusammenführung über 600.000 Menschen in beide deutsche Staaten, zwischen 1980 und 1999 waren es weitere 830.000 Personen, die meisten davon in den Jahren 1987 bis 1990. Sie alle wurden mit dem bestmöglichen Aufenthaltstitel in der Bundesrepublik aufgenommen und nach kurzer Zeit eingebürgert. Dadurch ergaben sich für die Betroffenen weitgehende politische, aber auch soziale Rechte. Dazu zählten die Anerkennung der Berufsausbildung, der sofortige Zugang zum Arbeitsmarkt sowie eine großzügige Integration in die deutschen Sozialversicherungssysteme. Eine andere Situation erwartete alle diejenigen, die keine Ansprüche auf die deutsche Staatsbürgerschaft erheben konnten, sondern als Flüchtlinge und Asylbewerber kamen. Aber auch sie konnten nach 1990 ihren Aufenthaltsstatus festigen und in Deutschland bleiben, viele wurden deutsche Staatsbürger.

Bei näherer Betrachtung der großen Aussiedlergruppe finden sich jedoch gravierende Unterschiede: Während die meisten Aussiedler bis etwa 1985 zumindest teilweise noch deutsch sozialisiert waren und heute nur noch wenige Kontakte zu ihrer alten Heimat pflegen, waren unter den Ankömmlingen der späten 1980er Jahre viele junge Menschen, die ohne Deutschkenntnisse einreisten und meistens nur polnisch sozialisiert waren. Sie entwickelten verschiedene Anpassungsstrategien an die neue Umgebung; viele von ihnen wurden »unsichtbar«. Aus dieser Gruppe rekrutieren sich heute Mitglieder und Vertreter der die polnische Identität betonenden »Polonia«-Organisationen.

Marek Wójcicki, der Chef des Bundes der Polen "Rodło", bezeichnet den Großteil der Migranten aus Polen eindimensional als "Polen«. In einem Interview in der Tageszeitung "Die Welt» vom 12. Januar 2010 sagte er: »Ich bin als Aussiedler gekommen, ich war im Aussiedlerheim. Aber ich bin Pole. Das war eine schizophrene Zeit: Man musste sich als Deutscher ausgeben, damit man in den Westen kam. Ich denke, mindestens eine Million dieser Aussiedler fühlen sich als Polen."

Diese Wahrnehmung teilen die Vertreter der "Polonia«-Organisationen mit konservativen Politikern sowie den Medien des nationalistischen politischen Spektrums in Polen, etwa aus dem Verlag von Pater Tadeusz Rydzyk (»Radio Maryja«, »Nasz Dziennik«, »Gazeta Polska»). Mit dem polnischen Anspruch auf die nationale Identität für die gesamte Gruppe (»bis zu 2 Mio.») ist gleichzeitig der Vorwurf an den deutschen Staat verbunden, der angeb- 
lich durch entsprechende Vorenthaltung der Minderheitenrechte »bewusst die Assimilierung polnischer Migranten fördere«. Dabei wurden nicht nur Stimmen von politischen Hardlinern laut; in den Sog der Forderungen nach Unterlassung assimilatorischer Praktiken gerieten auch Politiker, die man sonst nicht der Panikmache verdächtigen würde. So behauptete etwa auch der damalige Chef der Auslandspolen-Vereinigung "Wspólnota Polska«, Maciej Płażyński, in einem Beitrag in der Tageszeitung "Rzeczpospolita«, dass die Polen in Deutschland einer Politik nach der Devise "ein Reich, ein Volk» ausgesetzt seien: „Da hat sich seit Bismarcks Zeiten nichts geändert!"

Nur selten kommt es den polnischen Beteiligten in den Sinn, dass sie öffentlich einen Etikettenschwindel für sich reklamieren und von dem deutschen Partner dessen nachträgliche Billigung verlangen. Sicherlich sind Nuancen in der Wahrnehmung der Identitäten innerhalb der heterogenen Gruppe der Migranten aus Polen in Deutschland wichtig (»hybride Identitäten«). Dennoch fällt es deutschen Politikern schwer zuzugeben, in der Einwanderungspolitik gegenüber Spätaussiedlern aus Polen Fehler gemacht zu haben. Andernfalls müsste es ja heißen, dass eine große Gruppe von damaligen Einwanderern bei den Anerkennungsverfahren gelogen hätte, um sich durch die (verhältnismäßig einfache) Einbeziehung in den Staatsverband und das Sozialsystem der Bundesrepublik politische und materielle Rechte zu erschleichen. Zu einfach scheint die Argumentation der "Polonia«-Aktivisten zu sein, man müsse diese Lage heute widerspruchslos akzeptieren. Diese Einstellung verkennt die emotionale und materielle Leistung der deutschen Politik wie der Gesellschaft, die jahrzehntelang die Einwanderung der »Deutschstämmigen« aus dem Osten als Folgen des Krieges akzeptierte. Nun stellen sich "Deutschstämmige« aber in der Rhetorik der »Polonia" als »Polnischstämmige« heraus. Ihre Vertreter verlangen so vom deutschen Staat, doppelt »bedient« zu werden: Zunächst hatte sich dieser verpflichtet, Deutschkurse für die Betroffenen zu organisieren, weil diese bei ihrer Einreise angaben, an der Weitergabe der deutschen Sprache innerhalb und außerhalb der Familie durch die Maßnahmen des (kommunistischen - im Sinne »deutschfeindlichen«) polnischen Staates behindert worden zu sein. Heute will man aber denselben Staat in die Pflicht nehmen, die Förderung der polnischen (Mutter)Sprache und Kultur für dieselben Menschen und/oder deren Nachkommen staatlich zu organisieren und zu finanzieren und dieses Recht als flächendeckend einklagbar zu definieren. Dieser Aspekt entkräftet den Vorwurf, die deutsche Seite mache sich in der Minderheitenfrage selbst zur »Geisel ihres Rechtsstandpunkts«; es erklärt auch ein wenig die deutsche Befindlichkeit, die offiziell nicht immer klar zum Ausdruck kommt.
Dabei entsteht eine komplexe, ja schizophrene Situation: Der deutsch-polnische Vertrag meint zwar zwei verschiedene Gruppen von Menschen, die beim näheren Hinsehen aber austauschbar sind. Angehörige der deutschen Minderheit in Polen können - theoretisch wie praktisch - in die Bundesrepublik einreisen und hier in den Genuss der Regelungen für die polnischsprachige Gruppe kommen und umgekehrt: Deutsche Staatsbürger polnischer Herkunft können in Polen den Status eines Angehörigen der deutschen Minderheit für sich beanspruchen. Dies führt zu kuriosen Situationen: Während im Fussballbereich Wechselbeziehungen als Beispiele unproblematischer deutsch-polnischer Gegenwart aufgefasst werden (Lukas Podolski, Sebastian Boenisch, Eugen Polanski), wird andererseits die durch einen gewonnenen Prozess (bezüglich Eigentumsrückgabe) in ganz Polen bekannte Agnes Trawny in der polnischen öffentlichen Diskussion ausschließlich als "Deutsche« bezeichnet. Dabei müssten alle diejenigen, die von 2 Mio. Polen in Deutschland sprechen, Frau Trawny konsequenterweise als Polin betrachten, da sie vor einigen Jahrzehnten als Spätaussiedlerin nach Deutschland ging.

Bei aller Anspruchs- und Abwehrhaltung der offiziellen Gesprächspartner wird viel zu wenig auf die Betroffenen selbst geschaut. Nur ein kleiner Teil von ihnen ist überhaupt an der Arbeit der Organisationen der deutschen »Polonia« interessiert, geschweige denn aktiv. Die "Polonia« zählt zwar nach eigenen Angaben viele hundert Verbände, kann aber insgesamt nur auf wenige Mitglieder und Sympathisanten verweisen. Angesichts der ca. 1,5 Mio. Migranten aus Polen kann hier insgesamt nur von ein paar Hundert »Aktiven« in den Organisationen der »Polonia" gesprochen werden. Die früher dominierende Zerstrittenheit und die bis heute diffuse, da immer wieder die "Aufrechterhaltung der polnischen Identität» beschwörende Rhetorik ihrer Organisationen erschreckten viele, die ihr »Polnisch-Sein« auf eigene Art leben wollen.

Es bleibt noch einmal festzuhalten: Ein Großteil der Betroffenen ist an der politischen Tätigkeit der "Polonia» nicht interessiert; die stark deutsch sozialisierten Aussiedler haben bewusst ihre Brücken nach Polen abgebrochen, andere zeigen erfahrungsgemäß kein Interesse an deutsch-polnischen oder polnischen Belangen. Auch die viel beschworene "Generation Podolski« - die Kinder der in den 1970er und 1980er Jahren eingewanderten Aussiedler - erweist sich weitgehend als ein Phantom. Ihre Vertreter wuchsen zumeist ohne polnische Sprachkenntnisse auf und integrierten sich vollständig in die deutsche Gesellschaft; auf die wenigen Ausnahmen, die Menschen mit doppelter Identität, wird zwar in den Medien oft hingewiesen, ihre Zahl bleibt jedoch gering. 
Zwar beklagen viele der betroffenen Spätaussiedler in Literatur, Lebensberichten und Interviews, dass sie sich nach der Ankunft in Deutschland einem enormen Anpassungsdruck ausgesetzt gefühlt hätten, den deutsche Behörden, die Schule oder Nachbarn auf sie ausübten. So haben sie es unterlassen, den polnischen Teil ihrer Biographie zu pflegen bzw. an ihre Kinder weiterzugeben. Diese Aussagen begründen u. a. die unter »Polonia«-Funktionären verbreitete These vom Interesse der deutschen Politik an der Assimilierung der polnischen Migranten. Bei näherer Betrachtung lassen sich die Ängste und Bedenken der Betroffenen zum Zeitpunkt ihrer Ankunft in Deutschland nicht direkt auf äußere Einwirkungen zurückführen. Sie waren sicher vorhanden, glichen aber mehr einem selbsterzeugten Druck, die bisherige Identität möglichst schnell zu verändern oder anzupassen (Name, Sprache, soziale Kontakte).

Der Glaube an die hohe Zahl der in Deutschland lebenden nationalbewussten "Polen" ist demnach seitens der "Polonia«-Funktionäre als Manipulation und seitens einiger polnischer Politiker bestenfalls als Naivität zu werten. Dabei ist es keineswegs klar, wen die "Polonia« eigentlich repräsentiert. Zum Vergleich: Auf eine viel breitere Basis können die über 7.000 Mitglieder der überall in Deutschland aktiven deutsch-polnischen Gesellschaften blicken, deren Landes- und Ortsverbände programmatisch dialogbetonte (deutschpolnische, manchmal auch multikulturell definierte) Aktivitäten aufweisen. Angesichts der in die Millionen gehenden Zahl von Migranten aus Polen sind aber auch diese Gesellschaften eher eine Randerscheinung.

\section{Wie geht es mit der "Polonia" weiter?}

Aus heutiger Sicht kann durchaus von einem Erfolg der »Polonia«-Organisationen am Runden Tisch 20102011 gesprochen werden: Die von deutscher Seite unternommenen Schritte und zugesicherten Mittel werden die beschworene »Asymmetrie« im Umgang mit beiden Volksgruppen in beiden Ländern verringern. Auch die in der deutschen Politik zu spürende »neue Sensibilität» wird womöglich die Atmosphäre entspannen.

Die deutsche Seite kann ihrerseits einen Zeitplan entwickeln, wie und wann die angekündigten Maßnahmen für die polnische Gruppe realisiert werden. Von besonderer Brisanz sollten dabei die organisatorische Unterstützung und finanzielle Förderung des polnischen muttersprachlichen Unterrichts sein. Auf die Notwendigkeit eines größeren Engagements der deut- schen Schulbehörden in diesem Bereich wies bereits vor Jahren der deutsch-polnische Gesprächskreis »Kopernikus-Gruppe « hin und zwar mit Blick auf das Erlernen des Polnischen als Mutter- und als Fremdsprache (s. Quelle 4). Heute steht die Forderung der »Polonia«Organisationen im Raum, dass der Unterricht dann flächendeckend zu gewährleisten sei, wenn mindestens sieben Kinder an einer Schule dies verlangen. Die Gestaltung der Bildungsangebote obliegt hier den für Kultusaufgaben zuständigen Ländern, der Bund sollte die Sache jedoch als Priorität betrachten und die Länder finanziell unterstützen. Für den Anfang würde es sicherlich reichen, wenn in ausgewählten Schulen größerer Städte Polnisch als Muttersprache angeboten würde. Um die Kapazitäten zu nutzen und Synergieeffekte zu erzielen, sollte zugleich darüber nachgedacht werden, wie in den betroffenen Schulen/Städten Polnisch auch als Fremdsprache in den Fächerkanon der öffentlichen Schulen aufgenommen werden könnte (ein gymnasiales Lehrwerk wurde 2009 auf den Markt gebracht). Dies würde die Wahrnehmung der Sprache des Nachbarn erhöhen und ihre Anerkennung durch deutsche Schülerinnen und Schüler stärken. Hier sind mittlerweile auch erste Ergebnisse - etwa in Hessen - vorzuweisen.

In der Pflicht sind nun auch die »Polonia«-Aktivisten. Allerdings reicht es nicht, die Homepage einem »Lifting" zu unterziehen, um sich ein neues Image zu verpassen, denn hinter den Hochglanzbildern stehen immer noch oft die alten nationalbetonten Ideen. Es müssen neue Inhalte und gleichzeitig ein neues Bewusstsein geschaffen werden, die die Situation der in Deutschland lebenden "polnischen Gruppe" in moderneren Kategorien beschreiben. Mit der Aufwertung polnischen Lebens hierzulande, unterstrichen durch die Entschließung des Bundestags, kommt den Organisationen der "Polonia» eine neue Rolle zu - zu beweisen, dass (höhere) Finanzmittel nicht nur gefordert, sondern auch sinnvoll angelegt werden können. Bisher taten sich ihre Repräsentanten nicht mit Ideen hervor, die die "polnischen Massen" und die deutschen Zuwendungsgeber besonders beeindruckt hätten und gleichzeitig auch für die deutsche Mehrheitsgesellschaft attraktiv wären. Das müsste sich ändern, wollen die Organisationen der Polen in Deutschland glaubwürdig sein. Denn auch ohne den Minderheitenstatus kann man - das beweisen die nach Außen viel aktiveren deutsch-polnischen Gesellschaften - kulturell, gesellschaftlich und politisch etwas bewegen. 


\section{Quellen zum Text}

\section{Quelle 1:}

Antrag der Fraktionen CDU/CSU, SPD, FDP und BÜNDNIS 90/DIE GRÜNEN, Deutscher Bundestag, 17. Wahlperiode, Drucksache 17/6145 vom 9.6.2011

\section{Deutschland und Polen - Verantwortung aus der Geschichte, Zukunft in Europa}

Der Bundestag wolle beschließen:

I. Der Deutsche Bundestag stellt fest:

(...)

Polen und Deutschland betrachten heute die deutsche Minderheit in Polen und die polnischstämmigen Bürger in Deutschland als natürliche Brücken der Verständigung zwischen dem deutschen und dem polnischen Volk.

Viele Menschen mit polnischen Wurzeln wurden im Laufe der Geschichte zu Deutschen und haben aktiven Anteil an der gesellschaftlichen und kulturellen Entwicklung unseres Landes. Noch heute zeugen viele Familiennamen davon. In der Zeit des Nationalsozialismus aber wurden Angehörige der damaligen polnischen Minderheit in Konzentrationslagern umgebracht, ihre Organisationen verboten und enteignet. Der Bundestag will diese Opfer ehren und rehabilitieren. Wir sprechen uns deshalb für die Einrichtung einer Dokumentationsstelle zur Geschichte und Kultur der Polen in Deutschland aus. Wir bekräftigen zudem die Rechte zur Stärkung der kulturellen und sprachlichen Identität und befürworten die Förderung der Arbeits- und Entwicklungsmöglichkeiten für die polnischstämmigen Bürger in Deutschland, einschließlich der Eröffnung eines Büros in Berlin. (...)

\section{Zusammenarbeit auf dem Gebiet von Kultur, Wissenschaft und zivilgesellschaftlichem Austausch}

(...)

Etwa 2,4 Millionen Polen lernen Deutsch. Deutsch ist damit nach Englisch die wichtigste Fremdsprache in Polen. Der Deutsche Bundestag befürwortet den Ausbau der Möglichkeiten zur Vermittlung der polnischen Sprache als Herkunfts- und Fremdsprache in Deutschland.

(...)

Wir wollen das bestehende Netz der engen zivilgesellschaftlichen und wissenschaftlichen Zusammenarbeit immer enger knüpfen und halten dafür besonders bei der Vermittlung der polnischen Sprache in Deutschland und beim Schüler- und Studentenaustausch weitere Anstrengungen für nötig. (...)

II. Der Deutsche Bundestag fordert die Bundesregierung auf:

(...)

- in enger Zusammenarbeit mit den Bundesländern das Interesse vor allem der jungen Generation in Deutschland $(\ldots)$ an der polnischen Sprache und Kultur zu fördern

Berlin, 15. Juni 2011

Quelle: http://dipbt.bundestag.de/dip21/btd/17/061/1706145.pdf 
Quelle 2:

\section{Übereinstimmende Erklärung der Deutschen und der Polnischen Regierung über den Schutz der beiderseitigen Minderheiten, veröffentlicht am 5. November 1937}

Die Deutsche Regierung und die Polnische Regierung haben Anlaß genommen, die Lage der deutschen Minderheit in Polen und der polnischen Minderheit in Deutschland zum Gegenstand einer freundschaftlichen Aussprache zu machen. Sie sind übereinstimmend der Überzeugung, daß die Behandlung dieser Minderheiten für die weitere Entwicklung der freundnachbarlichen Beziehungen zwischen Deutschland und Polen von großer Bedeutung ist und daß in jedem der beiden Länder das Wohlergehen der Minderheit um so sicherer gewährleistet werden kann, wenn die Gewißheit besteht, daß in dem anderen Land nach den gleichen Grundsätzen verfahren wird. Zu ihrer Genugtuung haben die beiden Regierungen deshalb feststellen können, daß jeder der beiden Staaten im Rahmen seiner Souveränität für die Behandlung der genannten Minderheiten nachstehende Grundsätze als maßgebend ansieht:

1. Die gegenseitige Achtung deutschen und polnischen Volkstums verbietet von selbst jeden Versuch, die Minderheit zwangsweise zu assimilieren, die Zugehörigkeit zur Minderheit in Frage zu stellen oder das Bekenntnis der Zugehörigkeit zur Minderheit zu behindern. Insbesondere wird auf die jugendlichen Angehörigen der Minderheit keinerlei Druck ausgeübt werden, um sie ihrer Zugehörigkeit zur Minderheit zu entfremden.

2. Die Angehörigen der Minderheit haben das Recht auf freien Gebrauch ihrer Sprache in Wort und Schrift sowohl in ihren persönlichen und wirtschaftlichen Beziehungen wie in der Presse und in öffentlichen Versammlungen. Den Angehörigen der Minderheit werden aus der Pflege ihrer Muttersprache und der Bräuche ihres Volkstums sowohl im öffentlichen wie im privaten Leben keine Nachteile erwachsen.

3. Das Recht der Angehörigen der Minderheit, sich zu Vereinigungen, auch zu solchen kultureller und wirtschaftlicher Art, zusammenzuschließen, wird gewährleistet.

4. Die Minderheit darf Schulen in ihrer Muttersprache erhalten und errichten. Auf kirchlichem Gebiet wird den Angehörigen der Minderheit die Pflege ihres religiösen Lebens in ihrer Muttersprache und die kirchliche Organisierung gewährt. In die bestehenden Beziehungen auf dem Gebiet des Bekenntnisses und der caritativen Betätigung wird nicht eingegriffen werden.

5. Die Angehörigen der Minderheit dürfen wegen ihrer Zugehörigkeit zur Minderheit in der Wahl oder bei der Ausübung eines Berufes oder einer wirtschaftlichen Tätigkeit nicht behindert oder benachteiligt werden. Sie genießen auf wirtschaftlichem Gebiet die gleichen Rechte wie die Angehörigen des Staatsvolkes, insbesondere hinsichtlich des Besitzes oder Erwerbs von Grundstücken.

Die vorstehenden Grundsätze sollen in keiner Weise die Pflicht der Angehörigen der Minderheit zur uneingeschränkten Loyalität gegenüber dem Staat, dem sie angehören, berühren. Sie sind in dem Bestreben festgesetzt worden, der Minderheit gerechte Daseinsverhältnisse und ein harmonisches Zusammenleben mit dem Staatsvolk zu gewährleisten, was zur fortschreitenden Festigung des freundnachbarlichen Verhältnisses zwischen Deutschland und Polen beitragen wird. Quelle: http://clarysmith.com/scriptorium/deutsch/archiv/dokuvorgeschichte/dvk13.html\#101

\section{Quelle 3:}

Reichsgesetzblatt, Jahrgang 1940, Teil I (S. 444)

Verordnung über die Organisationen der polnischen Volksgruppe im Deutschen Reich.

\section{Vom 27. Februar 1940}

\section{$\$ 1$}

- Die Tätigkeit der Organisationen der polnischen Volksgruppe im Deutschen Reich (Vereine, Stiftungen, Gesellschaften, Genossenschaften und sonstige Unternehmen) ist verboten. Neue Organisationen der polnischen Volksgruppe dürfen nicht gegründet werden. 
- Die bisherigen Verwaltungsträger der Organisationen der polnischen Volksgruppe scheiden aus ihrem Amt aus. Sie können nicht über die Unternehmen der Organisationen und über diejenigen Vermögenswerte, die in einem rechtlichen oder wirtschaftlichen Zusammenhang mit dem Unternehmen stehen, verfügen.

- Ob eine Organisation als Organisation der polnischen Volksgruppe anzusehen ist, entscheidet im Zweifel der Reichsminister des Inneren.

$\$ 2$

- Der Reichsminister des Inneren wird ermächtigt, einen Kommissar für die Organisationen der polnischen Volksgruppen zu bestellen.

- Der Kommissar übt seine Tätigkeit nach den Weisungen des Reichsministers des Inneren aus und untersteht dessen Dienstaufsicht, er kann seine Befugnisse im Einzelfall übertragen.

$\$ 3$

- Der Kommissar führt die Verwaltung der Organisationen der polnischen Volksgruppe mit dem Ziel ihrer Liquidation und ist befugt, mit Wirkung für und gegen die Organisation zu handeln.

- Der Kommissar ist befugt, die Organisationen der polnischen Volksgruppe aufzulösen.

- Aufgelöste Organisationen der polnischen Volksgruppe sind vom Kommissar abzuwickeln. Der Reichsminister des Inneren kann im Einvernehmen mit dem Reichsminister der Justiz Richtlinien für die Abwicklung erlassen. In diesen Richtlinien kann von den allgemeinen Vorschriften über die Abwicklung abgewichen werden.

- Der Kommissar ist auf seinen Antrag bei Organisationen, die in öffentliche Register eingetragen sind, in das Register einzutragen.

$\$ 4$

- Der Kommissar ist nicht an Bestimmungen der Satzung oder Beschlüsse der Mitgliederversammlung (Gesellschaftsversammlung) einer Organisation gebunden, durch die die Geschäftsführung der Verwaltungsträger oder die Vermögensbewertung geregelt ist.

$\$ 5$

- Aus den auf Grund dieser Verordnung getroffenen Maßnahmen können Schadenersatzansprüche nicht abgeleitet werden.

$\$ 6$

- Wer sich entgegen dem $₫ 1$ an der Fortsetzung oder Neugründung einer Organisation der polnischen Volksgruppe beteiligt, wird mit Gefängnis und Geldstrafe oder einer dieser Strafen bestraft.

$\$ 7$

- Der Reichsminister des Inneren erlässt die zur Durchführung und Ergänzung dieser Verordnung erforderlichen Rechts- und Verwaltungsvorschriften.

\section{$\$ 8$}

- Die Inkraftsetzung dieser Verordnung für die eingegliederten Ostgebiete einschließlich des Gebiets der bisherigen Freien Stadt Danzig sowie für das Protektorat Böhmen und Mähren bleibt vorbehalten.

Berlin, den 27. Februar 1940.

Der Vorsitzende des Ministerrats für die Reichsverteidigung

Göring

Generalfeldmarschall

Der Generalbevollmächtigte für die Reichsverwaltung

Frick

Der Reichsminister und Chef der Reichskanzlei

Dr. Lammers 


\section{Quelle 4}

\section{Arbeitspapier III der Kopernikus-Gruppe Zur Frage der Förderung der polnischsprachigen Gruppe in Deutschland (Auszug)}

(September 2001)

\section{(...)}

In Deutschland wohnen ca. 1,5 Mio. polnischsprachige Menschen - in dem deutsch-polnischen Partnerschaftsvertrag vom 17. Juni 1991 bezeichnet als "Personen deutscher Staatsangehörigkeit in der Bundesrepublik Deutschland, die polnischer Abstammung sind oder sich zur polnischen Sprache, Kultur und Tradition bekennen« (Art. 20). Diese Menschen könnten und sollten eine wichtige Mittler- und Brückenrolle in dem deutsch-polnischen Dialog spielen. Leider ist es nicht so, und dies ist eine der im letzten Jahrzehnt ungenutzten Chancen. Dieser Zustand, der keine Seite zufrieden stellen kann, wird von mehren Faktoren bestimmt. (...)

Im wesentlichen besteht die Gruppe der 1,5 Mio. Polnischsprachigen aus den über 1 Mio. Personen, die in den 70er und 80er Jahren als Spätaussiedler zugewandert sind, also aus Personen, die sich unabhängig von einem komplizierten Zugehörigkeitsgefühl als Deutsche erklärt haben. Einen sehr wichtigen Teil dieser Gruppe bilden die Oberschlesier. Das sind zwar in ihrer großen Mehrheit nach ihrem Selbstverständnis keine Polen, aber mit Sicherheit Menschen, die mit der polnischen Sprache und Kultur verbunden sind, teilweise sogar Nachkommen der einstigen polnischen Minderheit im Deutschen Reich, die nach dem Zweiten Weltkrieg als Folge polnischer Politik der Zwangsassimilierung zur deutschen Minderheit mutierten. (...)

Ziel einer deutsch-polnischen Partnerschaft in Bezug auf die polnischsprachige Gruppe in Deutschland sollte es sein, die komplexe kulturelle Identität dieser Menschen zu bewahren. Natürlich bleibt die Frage der Identitätsfindung eine primär private Angelegenheit. Andererseits ist der Schlüssel zur Wahrung multikultureller Identität die Pflege der Mehrsprachigkeit. Dieses ist ein zentrales Problem des Schulwesens, der Bildungspolitik und somit des Staates. Zwei offene im Integrationsprozeß befindliche Gesellschaften sollten daran interessiert sein, dieses bikulturelle Potential der in Deutschland lebenden polnischsprachigen Menschen zu sichern.

Personen, die mit der polnischen Kultur verbunden sind, sollte die Möglichkeit gegeben werden, ihre Sprach- und Kulturkompetenz nutzbar zu machen. Diese Gemeinschaft sollte als natürliche Nachbarschaft, als Deutsche betrachtet werden, die etwas Besonderes über die polnischen, bis jetzt so wenig bekannten Nachbarn, zu sagen haben. Dies würde gewiß zum Überwinden von vielen der immer noch spukenden Stereotypen beitragen. (...)

Den Schlüssel zur Vermittlung der polnischen Kultur könnte ein Programm zum Erlernen der polnischen Sprache darstellen, und zwar für Polnisch als Muttersprache und als Fremdsprache. Bisher gibt es zwei isolierte und begrenzte Experimente in Bremen und Nordrhein-Westfalen. Polnisch sollte aber im deutschen Schulsystem breit und systematisch angeboten werden. Eine größere Zahl von Polnischlehrern würde ein natürliches, die polnische Kultur und die Kontakte mit Polen förderndes Milieu bilden. Die Schule könnte dann ausgebildete Polonisten aufnehmen, die heutzutage oft umsonst Ausschau nach einer Einstellung in Deutschland halten. Der Bedarf an ausgebildeten Polnischlehrern könnte auch ein Impuls zur Belebung der Polonistik-Studien an deutschen Hochschulen sein. Es wird angeregt, dass der polnische Staat mit Unterstützung polnischer und deutscher privater Träger an deutschen Universitäten Lehrstühle für »Polish Studies« stiftet.

(...)

Quelle: http://www.deutsches-polen-institut.de/Projekte/Projekte-Aktuell/Kopernikus-Gruppe/dritte-sitzung.php 


\section{Vom 18. bis zum 31. Oktober 2011}

\begin{tabular}{|c|c|}
\hline 18.10.2011 & $\begin{array}{l}\text { In einem Gespräch mit der Tageszeitung »Rzeczpospolita« bezeichnet der Parteivorsitzende von Recht und } \\
\text { Gerechtigkeit (Prawo i Sprawiedliwość - PiS), Jarosław Kaczyński, seine Äußerungen über Bundeskanzlerin } \\
\text { Angela Merkel in seiner vor den Parlamentswahlen Anfang Oktober erschienenen Publikation als Fehler, der } \\
\text { von den Medien ausgenutzt worden sei und zum schlechten Abschneiden von PiS bei den Parlamentswahlen } \\
\text { beigetragen habe. }\end{array}$ \\
\hline 19.10.2011 & $\begin{array}{l}\text { Leszek Miller, vor } 1989 \text { hoher Funktionär der Polnischen Vereinigten Arbeiterpartei (Polska Zjednoczona Par- } \\
\text { tia Robotnicza - PZPR) und 2001-2004 Ministerpräsident, wird zum neuen Fraktionsvorsitzenden der Demo- } \\
\text { kratischen Linksallianz (Sojusz Lewicy Demokratycznej-SLD) gewählt. Die SLD ist mit } 27 \text { Sitzen die kleinste } \\
\text { Fraktion im neu gewählten Sejm. }\end{array}$ \\
\hline 20.11.2011 & $\begin{array}{l}\text { Nach seiner Wahlniederlage zum Fraktionsvorsitzenden der Demokratischen Linksallianz (Sojusz Lewicy Demo- } \\
\text { kratycznej-SLD) am Vortag kündigt Ryszard Kalisz an, nicht für das Amt des Parteivorsitzenden zu kandidieren. }\end{array}$ \\
\hline 21.10 .2011 & $\begin{array}{l}\text { Ministerpräsident Donald Tusk trifft sich zu einem Gespräch mit dem Fraktionsvorsitzenden der Demokrati- } \\
\text { schen Linksallianz (Sojusz Lewicy Demokratycznej - SLD), Leszek Miller. Miller bezeichnet dieses als höfli- } \\
\text { che Geste und weist Gerüchte zurück, dass es sich um ein Koalitionsgespräch gehandelt habe. Es seien organi- } \\
\text { satorische Angelegenheiten der parlamentarischen Arbeit besprochen worden. }\end{array}$ \\
\hline 22.10 .2011 & $\begin{array}{l}\text { Der Fraktionsvorsitzende der Demokratischen Linksallianz (Sojusz Lewicy Demokratycznej - SLD), Leszek } \\
\text { Miller, kündigt an, dass die SLD in der neuen Legislaturperiode rasch einen neuen Entwurf zum Lebenspart- } \\
\text { nerschaftsgesetz einbringen wird, der sowohl gleichgeschlechtliche als auch nicht gleichgeschlechtliche Part- } \\
\text { nerschaften umfassen und Aspekte wie gemeinsames Eigentum, Besteuerung, Erbschaft und Rentenbezüge } \\
\text { berücksichtigen soll. Die SLD sei dabei offen für die Zusammenarbeit mit der Palikot-Bewegung (Ruch Pali- } \\
\text { kota) und der Bürgerplattform (Platforma Obywatelska - PO). }\end{array}$ \\
\hline 23.10 .2011 & $\begin{array}{l}\text { Ministerpräsident Donald Tusk (Bürgerplattform/Platforma Obywatelska - PO) unterstreicht, dass er keine } \\
\text { Probleme sehe, erneut eine Koalition mit der Polnischen Bauernpartei (Polskie Stronnictwo Ludowe - PSL) } \\
\text { einzugehen. }\end{array}$ \\
\hline 24.10 .2011 & $\begin{array}{l}\text { In einem Interview für die Tageszeitung »Nasz Dziennik«spricht sich der stellvertretende Vorsitzende von Recht } \\
\text { und Gerechtigkeit (Prawo i Sprawiedliwość - PiS) und ehemalige Justizminister Zbigniew Ziobro für eine } \\
\text { Demokratisierung und breite Öffnung der Partei aus. Gleichzeitig müsse die Einheit der Partei gewahrt wer- } \\
\text { den, was insbesondere unter dem Vorsitz von Jarosław Kaczyński gewährleistet werden könne. Ähnlich hatte } \\
\text { er sich gegenüber der Wochenzeitung »Uważam Rze« geäußert. }\end{array}$ \\
\hline 25.10 .201 & $\begin{array}{l}\text { Nach neuesten Angaben des Statistischen Hauptamts (Główny Urząd Statystyczny - GUS) betrug die Arbeits- } \\
\text { losenrate im September wie im August 11,8 \%. Im September } 2010 \text { hatte die Quote 11,5\% betragen. }\end{array}$ \\
\hline 25.10 .2011 & $\begin{array}{l}\text { Mariusz Błaszczak, erneut gewählter Fraktionschef von Recht und Gerechtigkeit (Prawo i Sprawiedliwość - } \\
\text { PiS), kündigt an, dass der Beauftragte für Disziplinarmaßnahmen in der Partei die jüngsten Äußerungen von } \\
\text { Zbigniew Ziobro, stellvertretender Vorsitzender von PiS, bewerten wird. Ziobro hatte sich wiederholt öffent- } \\
\text { lich für eine Demokratisierung in der Partei ausgesprochen. }\end{array}$ \\
\hline 26.10 .2011 & $\begin{array}{l}\text { Ministerpräsident Donald Tusk teilt in Brüssel im Rahmen der EU-Ratspräsidentschaft Polens mit, dass der } \\
\text { Europäische Rat zu einer Einigung über die Rekapitalisierung der Banken gekommen sei. Die Einigung trete } \\
\text { erst in Kraft, wenn sich die Länder der Eurogruppe über weitere krisenbekämpfende Maßnahmen geeinigt } \\
\text { haben. Tusk hebt hervor, dass sich die Zusammenarbeit innerhalb der Eurogruppe nicht gegen die Einheit der } \\
\text { Europäischen Union als ganzer wenden dürfe. }\end{array}$ \\
\hline 27.10 .2011 & $\begin{array}{l}\text { Fraktionsmitglieder der Palikot-Bewegung (Ruch Palikota) reichen beim Sejmmarschall einen Antrag auf Ent- } \\
\text { fernung des Kreuzes im Plenarsaal ein. Sollte diesem nicht stattgegeben werden, werde eine Beschwerde beim } \\
\text { Verfassungsgericht eingereicht, kündigt der Fraktionsvorsitzende Janusz Palikot an. }\end{array}$ \\
\hline 28.10 .2011 & $\begin{array}{l}\text { Der Parteivorsitzende der Polnischen Bauernpartei (Polskie Stronnictwo Ludowe - PSL), Waldemar Pawlak, } \\
\text { teilt mit, dass auf der ersten Sitzung des neu gewählten Sejm ein Gesetzesentwurf seiner Partei zur Kranken- } \\
\text { versicherung der Landwirte vorliegen wird. Der Vorschlag ist noch nicht mit der Bürgerplattform (Platforma } \\
\text { Obywatelska - PO) abgestimmt. Im vergangenen Jahr hatte das Verfassungsgericht geurteilt, dass die Finan- } \\
\text { zierung der Krankenversicherung für die Landwirte aus dem öffentlichen Haushalt nicht rechtskonform sei, } \\
\text { und dem Parlament für eine Gesetzesänderung } 15 \text { Monate Zeit eingeräumt. Bisher hatten PO und PSL noch } \\
\text { keine Einigung in dieser Sache erzielt. }\end{array}$ \\
\hline
\end{tabular}




\begin{tabular}{|l|l|}
\hline 29.10.2011 & $\begin{array}{l}\text { Auf der Sitzung des Landesrats (Rada Krajowa) der Demokratischen Linksallianz (Sojusz Lewicy Demokra- } \\
\text { tycznej - SLD) kündigt der Parteivorsitzende Grzegorz Napieralski an, sein Amt am 10. Dezember als Konse- } \\
\text { quenz aus der Wahlniederlage der SLD in den Parlamentswahlen niederzulegen. }\end{array}$ \\
\hline 30.10 .2011 & $\begin{array}{l}\text { Der Pressesprecher der Demokratischen Linksallianz (Sojusz Lewicy Demokratycznej - SLD), Tomasz Kalita, } \\
\text { gibt bekannt, dass ein Think Tank der Linken gegründet wird, der sich mit dem Wiederaufbau der Linken aus- } \\
\text { einandersetzen wird. Das Daszyński-Zentrum (Centrum Daszyńskiego) wird seinen Sitz in Warschau und in } \\
\text { Krakau haben; geschäftsführender Direktor soll Kalita werden. }\end{array}$ \\
\hline 31.10 .2011 & $\begin{array}{l}\text { In einem Interview für das Wochenmagazin »Der Spiegel« erklärt der Chef der Polnischen Nationalbank (Naro- } \\
\text { dowy Bank Polski - NBP), Marek Belka, dass der Euro gerettet werde und weiterhin stark sei. Andernfalls } \\
\text { würde die Idee der Europäischen Union zusammenbrechen, was für Polen eine Katastrophe bedeuten würde. } \\
\text { Aufgrund der derzeit schlechten Nachrichten über die gemeinsame Währung sei es aber gegenwärtig zu früh, } \\
\text { die Polen vom Nutzen der Einführung des Euro zu überzeugen. }\end{array}$ \\
\hline
\end{tabular}


Die Polen-Analysen erscheinen zweimal monatlich als E-Mail-Dienst. Sie werden gemeinsam vom Deutschen PolenInstitut Darmstadt, der Bremer Forschungsstelle Osteuropa und der Deutschen Gesellschaft für Osteuropakunde herausgegeben.

Ein Archiv der Polen-Analysen finden Sie im Internet unter www.laender-analysen.de/polen

Kostenloses Abonnement unter http://www.deutsches-polen-institut.de/Newsletter/subscribe.php

Diese Analysen finden Sie online als Lizenzausgabe auf

bpb.de

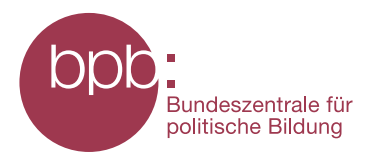

\section{Deutsches Polen-Institut Darmstadt}

Das Deutsche Polen-Institut Darmstadt (DPI) ist ein Forschungs-, Informations-, und Veranstaltungszentrum für polnische Kultur, Geschichte, Politik, Gesellschaft und die deutsch-polnischen Beziehungen, die sich im Kontext der europäischen Integration entwickeln. Das seit März 1980 aktive und bis 1997 von Gründungsdirektor Karl Dedecius geleitete Institut ist eine Gemeinschaftsgründung der Stadt Darmstadt, der Länder Hessen und Rheinland-Pfalz sowie des Bundes. 1987 wurden die Kultusminister der Länder und 2011 das Auswärtige Amt weitere institutionelle Träger. Einen wesentlichen Beitrag zur Verwirklichung der Institutsziele leisten private Stiftungen. Das DPI hat satzungsgemäß die Aufgabe, durch seine Arbeit zur Vertiefung der gegenseitigen Kenntnisse des kulturellen, geistigen und gesellschaftlichen Lebens von Polen und Deutschen beizutragen.

Ziel der Vermittlertätigkeit des DPI ist es, „die zu interessieren, auf die es politisch, wirtschaftlich, gesellschaftlich und kulturell im deutsch-polnischen Verhältnis ankommt (Leitlinien 1997). Es geht um die Entscheider und Multiplikatoren in Politik, Kultur, Bildung, Verwaltung, Medien und Wirtschaft und, wesentlich stärker ausgeprägt als bisher, um das Hineinwirken in Wissenschaft, Forschung und Bildung.

Derzeit bemüht sich das DPI in Kooperation mit den verstreuten Orten wissenschaftlicher Polen-Kompetenz an deutschen Hochschulen und Forschungsinstituten verstärkt darum, ausgehend von einer Bestandsaufnahme deutscher Polen-Forschung Ort wissenschaftlicher Forschung und verbindendes, vernetzendes und kooperierendes Zentrum zu werden. Ausgangspunkt der Neuausrichtung ist die kaum mehr kontrollierbare Dynamik des Rückbaus der Ressourcen der wissenschaftlichen Polen-Kompetenz in den unterschiedlichen Disziplinen. Mit der knapp 60.000 Bände zählenden multidisziplinären Fachbibliothek für Polen, die eine einzigartige Sammlung polnischer Literatur in der Originalsprache und in deutscher Übersetzung umfasst, ist das DPI bereits ein geschätzter Ort der Recherche und des wissenschaftlichen Arbeitens. (www.deutsches-polen-institut.de)

\section{Forschungsstelle Osteuropa an der Universität Bremen (www.forschungsstelle.uni-bremen.de)}

1982 gegründet, widmet sich die Forschungsstelle Osteuropa an der Universität Bremen der interdisziplinären Analyse der Länder Ost- und Ostmitteleuropas in Zeitgeschichte und Gegenwart. Der Forschungsschwerpunkt liegt dabei auf der Rolle von »Dissens und Konsens«, von Opposition und Zivilgesellschaft in ihrem historischen, politischen, gesellschaftlichen und kulturellen Kontext. Die Forschungsstelle besitzt in ihrem Archiv eine einzigartige Sammlung alternativer Kulturgüter und unabhängiger Texte aus den ehemaligen sozialistischen Ländern. Darunter befindet sich auch eine umfangreiche Sammlung des "Zweiten Umlaufs«, die das Schrifttum und Dokumente unabhängiger Initiativen und gesellschaftlicher Gruppen in Polen aus der Zeit von 1976 bis zum Umbruch umfasst. Hinzu kommt eine umfangreiche Bibliothek mit wissenschaftlicher Literatur. Mit Archiv, Bibliothek und zwei wissenschaftlichen Abteilungen ist die Forschungsstelle auch eine Anlaufstelle sowohl für Gastwissenschaftler als auch für die interessierte Öffentlichkeit.

Eine der Hauptaufgaben der Forschungsstelle ist die Information der interessierten Öffentlichkeit. Dazu gehören unter anderem regelmäßige E-Mail-Informationsdienste für Politik, Wirtschaft, Zivilgesellschaft und Medien. 